\title{
Changes in expression of the long non-coding RNA FMR4 associate with altered gene expression during differentiation of human neural precursor cells
}

\section{OPEN ACCESS}

Edited by:

William Cho,

Queen Elizabeth Hospital, Hong Kong

Reviewed by:

Francesco Nicassio,

Istituto Italiano di Tecnologia, Italy

Guney Bademci,

University of Miami, USA

Mohammadreza Hajjari,

Shahid Chamran University of Ahvaz,

Iran

*Correspondence:

Claes Wahlestedt,

Center for Therapeutic Innovation and Department of Psychiatry and Behavioral Sciences, Miller

School of Medicine, University of Miami, 1501 NW 10th Avenue, BRB \# 407 (M-860), Miami, FL 33136, USA cwahlestedt@med.miami.edu

Specialty section This article was submitted to

RNA

a section of the journal

Frontiers in Genetics

Received: 14 May 2015

Accepted: 27 July 2015

Published: 10 August 2015

Citation:

Peschansky VJ, Pastori C, Zeier Z, Motti D, Wentzel K, Velmeshev $D$, Magistri $M$, Bixby JL, Lemmon VP. Silva JP and Wahlestedt C (2015) Changes in expression of the long non-coding RNA FMR4 associate with altered gene expression during differentiation of human neural precursor cells.

Front. Genet. 6:263.

doi: 10.3389/fgene.2015.00263
Veronica J. Peschansky' ${ }^{1}$, Chiara Pastori' ${ }^{1}$, Zane Zeier ${ }^{1}$, Dario Motti ${ }^{2}$, Katya Wentzel' Dmitry Velmeshev ${ }^{1}$, Marco Magistri ${ }^{1}$, John L. Bixby ${ }^{2,3,4,5}$, Vance P. Lemmon ${ }^{2,3,4}$, José P. Silva ${ }^{1}$ and Claes Wahlestedt ${ }^{1 *}$

${ }^{1}$ Center for Therapeutic Innovation and Department of Psychiatry and Behavioral Sciences, Miller School of Medicine, University of Miami, Miami, FL, USA, ${ }^{2}$ Miami Project to Cure Paralysis, University of Miami, Miami, FL, USA, ${ }^{3}$ Department of Neurological Surgery, Miller School of Medicine, University of Miami, Miami, FL, USA, ${ }^{4}$ Center for Computational Science, University of Miami, Coral Gables, FL, USA, ${ }^{5}$ Department of Molecular and Cellular Pharmacology, Miller School of Medicine, University of Miami, Miami, FL, USA

CGG repeat expansions in the Fragile $X$ mental retardation 1 (FMR1) gene are responsible for a family of associated disorders characterized by either intellectual disability and autism Fragile $X$ Syndrome (FXS), or adult-onset neurodegeneration Fragile X-associated Tremor/Ataxia Syndrome. However, the FMR1 locus is complex and encodes several long non-coding RNAs, whose expression is altered by repeat expansion mutations. The role of these IncRNAs is thus far unknown; therefore we investigated the functionality of FMR4, which we previously identified. "Full"-length expansions of the FMR1 triplet repeat cause silencing of both FMR1 and FMR4, thus we are interested in potential loss-of-function that may add to phenotypic manifestation of FXS. Since the two transcripts do not exhibit cis-regulation of one another, we examined the potential for FMR4 to regulate target genes at distal genomic loci using gene expression microarrays. We identified FMR4-responsive genes, including the methyl-CpG-binding domain protein 4 (MBD4). Furthermore, we found that in differentiating human neural precursor cells, FMR4 expression is developmentally regulated in opposition to expression of both FMR1 (which is expected to share a bidirectional promoter with FMR4) and MBD4. We therefore propose that FMR4's function is as a gene-regulatory IncRNA and that this transcript may function in normal development. Closer examination of FMR4 increases our understanding of the role of regulatory IncRNA and the consequences of FMR1 repeat expansions.

Keywords: IncRNA, intellectual disability, epigenetics, differentiation, chromatin remodeling, MBD4, FMR4, Fragile $X$

Abbreviations: ASD, autism spectrum disorder; DIV, days in vitro; FMRP, Fragile X mental retardation protein; FXPOI, Fragile X-related primary ovarian insufficiency; FXS, Fragile X syndrome; FXTAS, Fragile X-associated tremor/ataxia syndrome; hNPCs, human neural precursor cells; hNS, human neurospheres; lncRNAs, long non-coding RNAs; mRNA, messenger RNA; ncRNAs, non-coding RNAs. 


\section{Introduction}

Fragile X Syndrome, FXTAS, and FXPOI are X-linked disorders that arise from expansions in a CGG-repeat region in the $5^{\prime}$-UTR of the FMR1 gene. Normal FMR1 alleles contain 654 repeats, expansions from 55 to 200 repeats are considered "premutations" and all larger repeat sizes are known as the "full mutations." Individuals with a premutation may develop the adult-onset neurodegenerative disorder known as FXTAS, while women carrying the premutation are at risk for FXPOI. Only the full mutation leads to FXS, which is a common cause of inherited intellectual disability and autism (Oostra and Willemsen, 2003). FMR1 premutations result in overproduction of toxic, expanded mRNAs that contribute to the development of FXPOI and FXTAS pathology (Tassone et al., 2000; Kenneson et al., 2001). Full mutations lead to DNA and repressive histone methylation of the FMR1 locus (Sutcliffe et al., 1992; Hornstra et al., 1993; Coffee et al., 1999, 2002; Kumari and Usdin, 2010). Thus FXS derives from the loss of FMR1 mRNA and protein FMRP. We and others have identified four non-coding transcripts with abnormal expression in response to Fragile $\mathrm{X}$ repeat expansions at the FMR1 locus (Ladd et al., 2007; Khalil et al., 2008; Pastori et al., 2014), but their role in FXS/FXTAS/FXPOI phenotypes remains to be determined.

The vast majority of the human transcriptome is comprised of either long [ $>200$ nucleotides (nt)] or short ncRNAs (Cheng et al., 2005; Banfai et al., 2012; Djebali et al., 2012). While short ncRNAs typically regulate gene expression through posttranscriptional mechanisms or by interfering with translation (Rother and Meister, 2011; Fabian and Sonenberg, 2012), lncRNAs (which can be many kilobases long) often act in cis or trans to regulate gene expression at their locus of origin or elsewhere in the genome, respectively. Evidence suggests that IncRNAs perform scaffolding functions by recruiting epigenetic complexes or ribonucleoproteins that cause chromatin remodeling (Wang and Chang, 2011). Other lncRNAs act posttranscriptionally by targeting mRNAs or translational machinery. Regardless of the mechanism, a growing body of evidence implicates lncRNAs in a myriad of normal cellular functions such as DNA damage response and mitosis (Tsai et al., 2010; Yap et al., 2010; Hung et al., 2011; Kotake et al., 2011; Wang and Chang, 2011) and in diseases, such as cancer (Hajjari et al., 2014).

Recent attention has focused more specifically on the role of $\operatorname{lncRNAs}$ in neurodevelopmental programs and diseases of the nervous system. For example, lncRNAs are involved in the differentiation of neural cell types, and synaptic signaling and maturation (Mercer et al., 2010; Qureshi et al., 2010). In addition, both short and long ncRNAs are known to be involved in Prader-Willi syndrome, which is a developmental disorder caused by paternal deletion of a maternally imprinted region and can present with metabolic dysregulation including circadian rhythm defects (Sahoo et al., 2008; De Smith et al., 2009; Powell et al., 2013). Both syndromic and non-syndromic ASD susceptibility loci also contain aberrantly expressed lncRNAs that may contribute to disease (Velmeshev et al., 2013; Ziats and Rennert, 2013). Dysfunction of lncRNAs has also been linked to pathogenesis of neurodegenerative disorders including Alzheimer's disease (Faghihi et al., 2008) and spinocerebellar ataxia type 7, another repeat expansion disorder (Sopher et al., 2011). In sum, there is a growing body of evidence supporting the involvement lncRNAs in both the normal and diseased nervous system, spurring further mechanistic inquiries.

$F M R 4$, an untranslated, antisense lncRNA originating at the $F M R 1$ gene locus was shown to have anti-apoptotic functions in HEK293T and HeLa cells but to have no effect on expression of FMR1 (Khalil et al., 2008). Here, we describe FMR4's function as a regulator of gene expression in trans by identifying mRNA expression changes induced by FMR4. In particular, these effects in HEK293T cells are mirrored by discordant developmental regulation between FMR4 and one of its targets, MBD4, in hNPCs.

\section{Materials and Methods}

\section{HEK293T Cell Culture, Transfection, and RNA Extraction}

HEK293T cells were cultured in DMEM with 10\% FBS. In overexpression experiments, cells were transfected with pcDNA3.1-FMR4 or the empty pcDNA3.1 control vector using Lipfectamine $2000 \mathrm{CD}$. For knockdown experiments, the siRNA FMR4(C) (Khalil et al., 2008), versus Silencer Negative Control siRNA \#1 (Ambion) were used with the Lipofectamine RNAiMAX transfection reagent, according to manufacturer's instructions (Invitrogen). For microarray experiments, $1 \times 10^{6}$ cells were plated and transfected with $0.5 \mu \mathrm{g}$ plasmid or $40 \mathrm{nM}$ siRNA on the same day, and incubated for 6 or $24 \mathrm{~h}$ after transfection. For validation, FMR4 was knocked down using two sequential siRNA transfections over $72 \mathrm{~h}$. RNA was extracted using Trizol (Invitrogen) and the RNeasy Mini Kit, and treated with DNAse on-column using the RNAse-free DNAse Set (Qiagen) according to manufacturer's instructions.

\section{Microarray Hybridization and Analysis}

At 6 or $24 \mathrm{~h}$ post transfection, RNA was extracted and samples were submitted to the Hussman Institute for Human Genomics Center for Genome Technology for microarray analysis using Affymetrix Human Gene 2.0 ST Arrays. Total RNA samples were first prepared using the Ambion WT Expression Kit (cat\# 4411974). Briefly, the kit generates sensestrand cDNA from total RNA using a reverse transcription priming method that specifically primes non-ribosomal RNA, including both poly(A) and non-poly(A) mRNA. Next, samples are fragmented and labeled using the Affymetrix GeneChip WT Terminal Labeling Kit (cat\# 902280). Final yield was hybridized onto the array, washed and stained using the Affymetrix GeneChip Hybridization, Wash, and Stain Kit (cat\# 900720). Arrays were scanned using GeneChip Scanner 3000 7G system. Background subtraction, GC-RMA normalization and quality control were performed using the Affymetrix GeneChip Command Console Software and the bioconductor package from R. Data have been archived in 
the Gene Expression Omnibus at the National Center for Biotechnology Information, and assigned the accession number GSE70817.

\section{cDNA Synthesis and Quantitative Real-Time PCR (qPCR)}

cDNA was synthesized using the High Capacity cDNA Reverse Transcription Kit (Applied Biosystems) with $500 \mathrm{ng}$ of total RNA per reaction. Gene-specific FMR4 cDNA was primed separately ("FMR4 RT": ATTGCTGGCAGTCGTTTCTT), in order to specifically detect the antisense transcript and prevent capture of overlapping sense transcripts arising from that genomic region. Random hexamer-primed cDNA libraries were used for detection of all other genes. FMR4 RNA expression was quantified using SYBR Green quantitative real-time PCR (qPCR) with the following primers, validated by melting curve: "FMR4 FW" - ACCAAACCAAACCAAACCAA and "FMR4 REV" - GTGGGAAATCAAATGCATCC. Commercially available TaqMan probes (Invitrogen) were used for all other transcripts (MBD4, cat\# Hs00187498_m1; FMR1, cat\# Hs00924547_m1; MALAT1, cat\# Hs01910177_s1). The endogenous control was glyceraldehyde 3-phosphate dehydrogenase (GAPDH, cat\# 4326317E) where necessary, and data were analyzed by the $\Delta \Delta \mathrm{Ct}$ method. For polyA detection, cDNA was synthesized from $1 \mu \mathrm{g}$ total RNA using the High Capacity cDNA kit and oligodT primers at $50 \mathrm{nM}$ final concentration. In the noRT control, we omitted the reverse-transcriptase enzyme from the cDNA synthesis reaction. Reverse transcription products were amplified using $\mathrm{qPCR}$ as described above and visualized on a $1.5 \%$ agarose gel.

\section{Human Neural Precursor Cell (hNPC) Culture, Differentiation, and Viral Transduction}

hNPCs used in this study were derived from human fetal brains collected from third trimester aborted fetuses received from the Birth Defects Research Lab at the University of Washington in Seattle. This work was classified as "NonHuman Subject Research" by the Human Subject Research Office at the University of Miami, and therefore was not subject to Institutional Review Board approval. Briefly, brain tissues were dissected and dissociated using the trypsin-based Neural Tissue Dissociation Kit (Miltenyi Biotec, cat \#130-093-231). Five million single cells were seeded into each $75-\mathrm{mm}$ tissue culture flask in proliferation media supplemented with B27 (1X of proprietary formula), human Epidermal Growth Factor (hEGF) at $20 \mathrm{ng} / \mathrm{mL}$, human Fibroblast Growth Factor (hFGF) at $10 \mathrm{ng} / \mathrm{mL}$, heparin at $20 \mathrm{ug} / \mathrm{mL}$, GlutaMax (1X, Gibco), and Primocin at $0.1 \mathrm{mg} / \mathrm{mL}$ (InvivoGen). Neural stem cells formed neurosphere colonies after approximately 7 DIV, while other cells remained in suspension as single cells or formed a monolayer on the flask surface. Neurospheres can be maintained as such in suspension for several months using proliferation media, or hNPCs can be differentiated, forming a mixture of neurons and astrocytes. Neurospheres were transduced with a lentiviral vector expressing FMR4 (pLentiCMV/TO-mCherryFMR4) or the control vector (pLentiCMV/TO-mCherry), and collected for qPCR analysis after 2 days. To differentiate, neurospheres are dissociated into single cells with Accutase and cultured in Advanced DMEM/F12 media without hFGF or hEGF, but with Bottenstein's N2 at $1 \mathrm{X}$ (Invitrogen, proprietary formula), $2.5 \%$ fetal bovine serum, heparin at $20 \mu \mathrm{g} / \mathrm{mL}$, GlutaMax (1X, Gibco) and Primocin at $0.1 \mathrm{mg} / \mathrm{mL}$ (InvivoGen).

\section{Subcellular Fractionation}

Neurospheres were collected by centrifugation at $250 \times g$ in order to form $50 \mu \mathrm{L}$ pellets. Pellets were washed with PBS and fractionated with the NE-PER Nuclear and Cytoplasmic Extraction Reagents (Thermo Scientific, cat\# 78835) according to manufacturer's instructions. Briefly, cells were sequentially lysed and centrifuged to first separate pelleted nuclei from cytoplasm, then to separate chromatin from nucleoplasmic components. RNA was extracted from solid chromatin with $1 \mathrm{~mL}$ of Trizol, and with $0.75 \mathrm{~mL}$ Trizol LS for every $0.25 \mathrm{~mL}$ of liquid fraction. cDNA prepared using random primers was used for TaqMan qPCR (MALAT1, GAPDH, and FMR1), while gene-specific priming and SYBR Green was used for FMR4 as noted above. In each case, starting material for cDNA reactions was $2 \mu \mathrm{L}$ total RNA (not equal mass of RNA), to enable comparison of absolute quantities of each transcript between compartments. Relative quantification (RQ) for each transcript in each compartment was calculated from $\mathrm{Cq}$ values by $\mathrm{qPCR}$. Individual fraction $\mathrm{RQ}$ values were normalized to the total detected amount for each transcript.

\section{Results}

\section{Fmr4 Induces Genome-Wide Changes in Gene Expression}

Previous studies of the $2.4 \mathrm{~kb}$ antisense lncRNA FMR4 described no cis-regulation of FMR1 (Khalil et al., 2008); therefore we hypothesized that FMR4 would regulate gene expression in trans, which is a well-documented function of other lncRNAs (Rinn et al., 2007; Kino et al., 2010; Miyagawa et al., 2012). In order to comprehensively measure gene regulation in response to FMR4 at the mRNA level, we treated HEK293T cells with either an siRNA against FMR4, a scrambled control siRNA (knockdown), pcDNA3.1-FMR4, or the empty pcDNA3.1 vector (overexpression) and processed for microarray hybridization after 6 or $24 \mathrm{~h}$. Using LIMMA, a linear modeling approach (Smyth, 2004), we identified differential expression of over 3,700 genes between FMR4 knockdown, overexpression and their respective control conditions, and characterized the pattern of target gene expression relative to FMR4. To this end, we used the Cluster Affinity tool of TIGR MultiExperiment Viewer to identify genes with opposite behavior in the knockdown condition compared to the overexpression condition. This strategy narrowed our focus to the 238 transcripts represented in Figure 1A and Supplementary Figure S1, which are further classified by whether they are concordant or discordant with respect to FMR4 changes. This analysis yielded 155 and 83 target genes with concordant and discordant changes in mRNA expression 
A

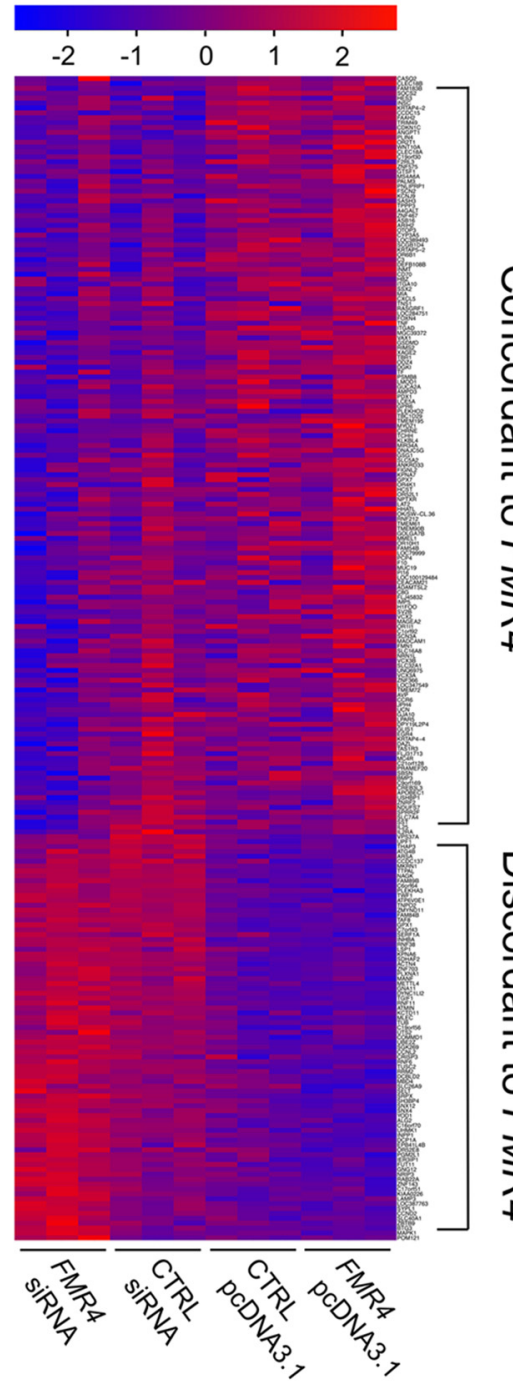

FIGURE 1 | Microarray analysis of mRNA expression changes in response to FMR4 knockdown and overexpression reveal

trans-regulatory targets. (A) Microarray analysis of HEK293T cells with FMR4 overexpression or knockdown $(n=3)$. LIMMA analysis and Cluster Affinity
C
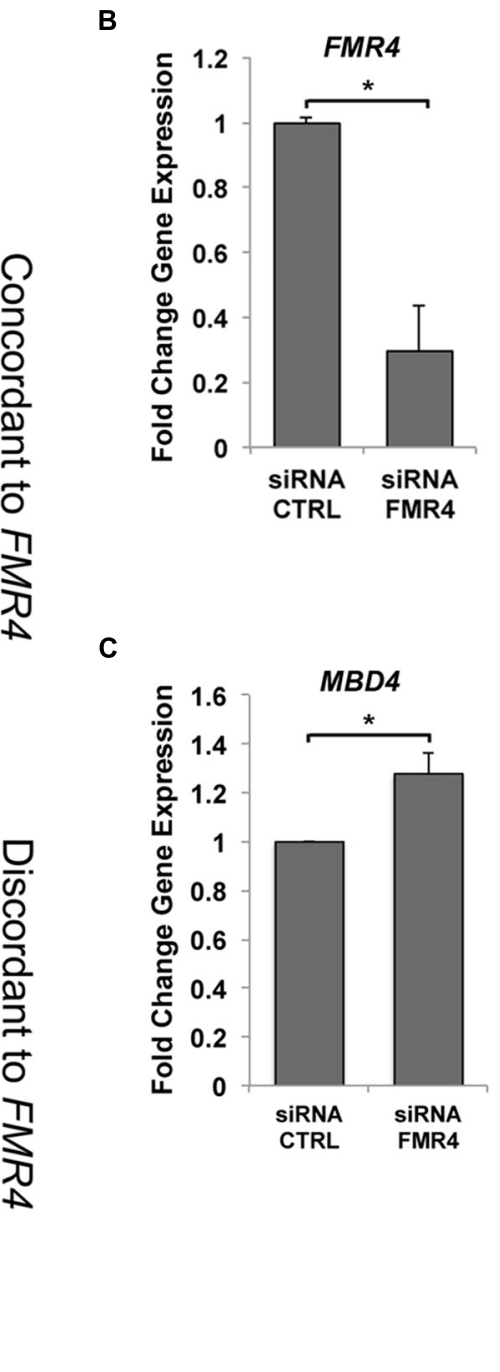

identified genes concordantly or discordantly regulated compared to FMR4. (B) FMR4 was knocked down with siRNA in HEK293T cells in order to validate changes in a putative FMR4-sensitive gene identified by microarray (C). ( $n=6$, ${ }^{*} p<0.05$ by Student's $t$-test). relative to FMR4, respectively. These data support the idea that FMR4 is a regulator of gene expression through transactivity.

\section{Pathway Analysis and FMR4 Target Validation}

We then analyzed the cohort of concordant and discordant FMR4-responsive genes with GeneGo Metacore (Tables 1 and 2). Cell cycle regulation and apoptosis were highly ranked biological processes affected by FMR4, which is consistent with our earlier findings (Khalil et al., 2008). Additionally, FMR4-sensitive genes are enriched in developmental processes in general and neurodevelopmental processes in particular (e.g., adrenergic and opioid signaling, Wnt pathway, cytoskeletal elements, synaptogenesis). Informed by the pathway analysis and previous insights into FMR4's function, we used HEK293T cells with FMR4 knocked down by sequential siRNA transfections (Figure 1B) to validate a $28 \%$ increase in methyl CpG-binding domain (MBD4; Figure 1C).

\section{Developmental Regulation of Gene Expression by FMR4 in hNPCs}

To investigate the putative role of FMR4 in neurodevelopment and Fragile X-associated neurological disorders, we used an in vitro model system consisting of human fetal-derived neurospheres (hNSs). These cells can be maintained as precursor in hNSs, or induced to differentiate into a mixed culture of early neurons and glia (see Materials and Methods). This system has the advantage of being a primary culture of human brain cells (critical for studying a primate-specific transcript in vitro) 
TABLE 1 | Top GeneGo pathways represented by FMR4-responsive genes identified by microarray analysis.

\begin{tabular}{|c|c|c|}
\hline Category & GeneGo pathway & $p$-value \\
\hline \multirow[t]{2}{*}{ Cell adhesion } & Chemokines and adhesion & 0.10161 \\
\hline & $\begin{array}{l}\text { Integrin-mediated cell adhesion and } \\
\text { migration }\end{array}$ & 0.11458 \\
\hline \multirow[t]{3}{*}{ Cell cycle } & Regulation of G1/S transition (part 2) & 0.00000 \\
\hline & $\begin{array}{l}\text { Nucleocytoplasmic transport of } \\
\text { CDK/Cyclins }\end{array}$ & 0.03480 \\
\hline & $\begin{array}{l}\text { Spindle assembly and chromosome } \\
\text { separation }\end{array}$ & 0.08019 \\
\hline \multirow[t]{9}{*}{ Development } & $\begin{array}{l}\text { Mu-type opioid receptor signaling via } \\
\text { beta-arrestin }\end{array}$ & 0.00000 \\
\hline & $\begin{array}{l}\text { S1P1 receptor signaling via } \\
\text { beta-arrestin }\end{array}$ & 0.00000 \\
\hline & $\begin{array}{l}\text { Beta-adrenergic receptors } \\
\text { transactivation of EGFR }\end{array}$ & 0.00000 \\
\hline & $\begin{array}{l}\text { Ligand-independent activation of ESR1 } \\
\text { and ESR2 }\end{array}$ & 0.00001 \\
\hline & Gastrin in cell growth and proliferation & 0.00002 \\
\hline & $\begin{array}{l}\text { Alpha-2 adrenergic receptor activation } \\
\text { of ERK }\end{array}$ & 0.00002 \\
\hline & $\begin{array}{l}\text { Regulation of } \\
\text { epithelial-to-mesenchymal transition } \\
\text { (EMT) }\end{array}$ & 0.04670 \\
\hline & $\begin{array}{l}\text { Lipoxin inhibitory action on PDGF, EGF, } \\
\text { and LTD4 signaling }\end{array}$ & 0.08717 \\
\hline & A3 receptor signaling & 0.11683 \\
\hline \multirow[t]{4}{*}{ Immune response } & $\begin{array}{l}\text { Oncostatin M signaling via MAPK in } \\
\text { mouse cells }\end{array}$ & 0.00000 \\
\hline & $\begin{array}{l}\text { Oncostatin M signaling via MAPK in } \\
\text { human cells }\end{array}$ & 0.00000 \\
\hline & IL-15 signaling & 0.00002 \\
\hline & $\begin{array}{l}\text { Histamine } \mathrm{H} 1 \text { receptor signaling in } \\
\text { immune response }\end{array}$ & 0.02758 \\
\hline Transport & RAN regulation pathway & 0.04453 \\
\hline $\mathrm{N} / \mathrm{A}$ & $\begin{array}{l}\text { Inhibitory action of Lipoxin A4 on PDGF, } \\
\text { EGF, and LTD4 signaling }\end{array}$ & 0.08485 \\
\hline
\end{tabular}

without the need for reprogramming, as would be the case with embryonic or induced pluripotent stem cells. We focused on the relationship between FMR4 and its putative target gene, $M B D 4$, which we identified by microarray analysis. MBD4 is a transcriptional repressor involved in DNA repair (Ballestar and Wolffe, 2001; Kondo et al., 2005). Expression of MBD4 developmentally regulates several tissues (Ruddock-D'cruz et al., 2008; Zhang et al., 2014a), and its aberrant expression in hippocampal GABAergic neurons in psychiatric disease may be linked to abnormal differentiation in these cells (Benes et al., 2009).

The FMR1 locus is crucial to normal brain development; thus, we wanted to determine whether FMR4 expression is dependent on developmental stage. We extracted RNA from undissociated hNS ["0 days in vitro" (DIV)] and cultured cells from dissociated hNS up to five DIV in differentiation media. At both time points, we measured FMR4 expression as well as that of FMR1, to determine whether these transcripts are independently regulated. We observed that FMR4 expression
TABLE 2 | Top GeneGo process networks represented by FMR4-responsive genes identified by microarray analysis.

\begin{tabular}{lll}
\hline Category & GeneGo process networks & $\boldsymbol{p}$-value \\
\hline Apoptosis & Death domain receptors and caspases & 0.02682 \\
in apoptosis & \\
Cell adhesion & Platelet aggregation & 0.02999 \\
Cell cycle & Leucocyte chemotaxis & 0.04076 \\
& G1/S Interleukin regulation & 0.00000 \\
Cytoskeleton & G1-S Growth factor regulation & 0.00002 \\
& Actin filaments & 0.00022 \\
Development & Actin filaments & 0.03088 \\
& Hemopoiesis, erythropoietin pathway & 0.00000 \\
& Neurogenesis and synaptogenesis & 0.08499 \\
& Neuromuscular junction & 0.11290 \\
DNA Damage & Wnt/beta-catenin, notch, VEGF, IP3, & 0.11674 \\
Immune response & and integrin signaling & \\
Inflammation & Checkpoint & 0.00006 \\
& BCR pathway & 0.00008 \\
& MIF signaling & 0.00000 \\
& IL-2 signaling & 0.00003 \\
Transduction & Protein C signaling & 0.06668 \\
\hline & Kallikrein-kinin system & 0.09179 \\
Reproduction & FSH-beta signaling pathway & 0.00001 \\
& CREM pathway & 0.00002 \\
& Neuropeptide signaling pathways & 0.01407 \\
\hline
\end{tabular}

is significantly decreased in differentiating cells at 5 DIV while FMR1 is increased at the same time point (Figure 2A). We then measured MBD4 and found that it is also developmentally regulated. As indicated by the microarray analysis, MBD4 was increased at a time point when FMR4 expression is low (Figure 2A). We also observed changes in expression level of other FMR4 target genes in undifferentiated, proliferating hNPCs transduced with an mCherry-tagged lentiviral vector expressing FMR4 (Supplementary Figure S2A). We found that FMR4 overexpression significantly upregulated two putative targets, the deubiquitinase YOD1 and the G-protein subunit GNG12, and downregulated the ribonucleotide reductase RRM2 (Supplementary Figure S2B). These data corroborate our finding that FMR4 regulates gene expression in trans.

\section{Molecular Mechanisms of FMR4}

To better understand the molecular role of FMR4, we performed subcellular fractionation of hNS. As expected, GAPDH and FMR1 mRNA were highest in the cytoplasmic fraction (87.6 and $67.0 \%$, respectively) where they are translated (Duszczyk et al., 2011) (Figure 2B). Some lncRNAs are expressed predominantly in the nucleus. A well-known example of this is the lncRNA NEAT2/MALAT1 (Gutschner et al., 2013). We measured this transcript as a positive control for nuclear transcripts, and found it enriched in the nucleoplasm (41.6\%) and chromatin (54.0\%) relative to the cytoplasm (Figure 2C). FMR4 RNA was primarily localized (73.4\%) to chromatin (Figure 2C), which is consistent with a transcriptional regulatory function of FMR4. 
A

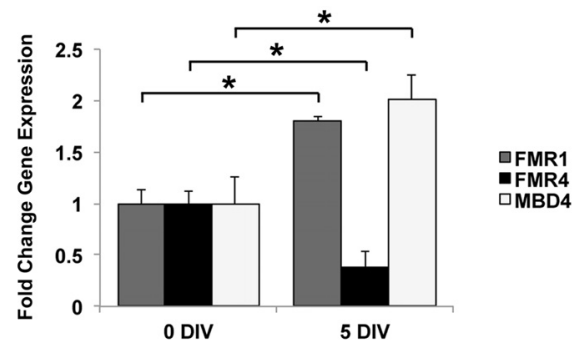

B

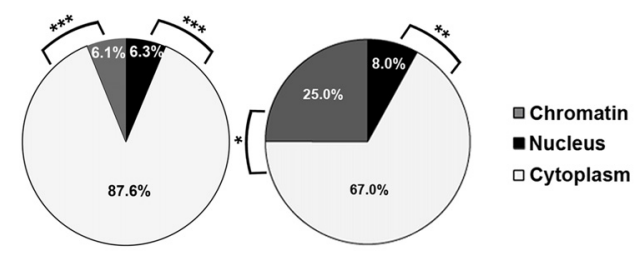

GAPDH

C

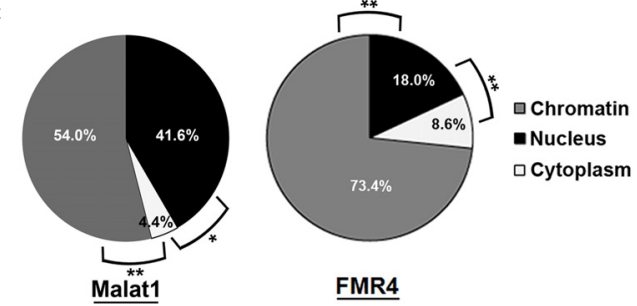

FIGURE 2 | Expression of FMR4 and its target MBD4 during differentiation of hNPCs may be related to chromatin-modulatory function of the IncRNA. (A) Comparison of RNA expression between proliferating neurospheres (0 DIV) and differentiating hNPCs (5 DIV) revealed increased FMR1 and decreased FMR4 after 5 days of differentiation $(n=5$, ${ }^{*} p<0.05$ by Student's $t$-test). As predicted, the putative FMR4 target gene MBD4 was upregulated with decreased FMR4. Subcellular fractionation of hNPCs with subsequent qPCR detection confirmed the cytoplasmic localization of mRNAs such as (B) GAPDH and FMR1. MALAT1 (C), known to be associated to nuclear speckles, was significantly enriched in the nucleus and chromatin compared to cytoplasm. FMR4 RNA (C) was largely localized to chromatin. ( $n=5,{ }^{*} p<0.05,{ }^{* *} p<0.01,{ }^{* * *} p<0.001$ by One-way ANOVA with Tukey HSD).

Since RNA polyadenylation frequently targets mature RNA for export into the cytoplasm (Zhang et al., 2014b); many lncRNAs are not polyadenylated. Therefore, to establish whether FMR4 can be polyadenylated, we performed first-strand cDNA synthesis with oligodT primers, thereby capturing polyadenylated transcripts from total hNS RNA. We observed via qPCR that in addition to FMR1 and GAPDH mRNAs (which are expected to be polyadenylated), a detectable portion of FMR4 is also polyadenylated (Supplementary Figure S3). These data suggest that a fraction of FMR4 may be stabilized by polyadenylation; this would increase the molecule's half-life and permit its diffusion to distant genomic loci.

\section{Discussion}

Loss of FMR1 mRNA and FMRP function has been widely studied, but there is relatively little known about ncRNA encoded by the locus. Here we show that one such ncRNA, FMR4, may regulate mRNA expression genome-wide via a developmentally regulated transcriptional mechanism, thereby impacting important biological processes.

Similar to FMR4, trans-acting lncRNAs [such as HOTAIR, MALAT1, and GAS5 (Rinn et al., 2007; Kino et al., 2010; Miyagawa et al., 2012)] affect loci far from their genomic locus of origin. In this study, we confirmed that knockdown and overexpression of FMR4 causes changes in genes involved in proliferation and differentiation. As a chromatin-associated transcript, FMR4 may act at the transcriptional level by forming complexes with histone modifying enzymes or by directly targeting mRNA stability, splicing, or editing. It remains to be seen whether these observations are dependent on RNA-protein interactions, and whether they result from direct epigenetic changes or via downstream effects.

Our data show that FMR4 is developmentally regulated in an hNPC model. After 5 days of in vitro differentiation, FMR4 expression is significantly reduced, while that of both FMR1 and the FMR4 target gene MBD4 is increased. Since $F M R 4$ and FMR1 do not interact in cis but are discordantly expressed with differentiation of hNPCs, the bidirectional promoter responsible for expression of both of these transcripts might be activated in only one direction at a time. An alternative possibility is that FMR4 RNA is degraded at a higher rate during this period while FMR1 is not. Nevertheless, it would be interesting to determine whether transcription factors normally regulate this locus as a whole or target each transcript individually. It is also unclear whether the decrease in FMR4 lncRNA expression contributes to disease in addition to loss of FMR1, or is an artifact of the locus-wide, full mutation-induced epigenetic changes causing transcriptional repression. Future studies will be necessary to distinguish between these possible mechanisms and consequences of FMR4 regulation.

Discordant regulation of MBD4 and FMR4 leads us to conclude that FMR4 regulates expression of genes at distal loci, as the same relationship was identified by our overexpression and knockdown studies. Our independent validation studies confirm this was not due to false positive identification, which is a common problem in genome-wide analyses such as microarray studies. FMR4's localization to the chromatin fraction points to a transcriptional mechanism for this effect, however, studies evaluating the direct binding of FMR4 to nucleic acids or proteins are warranted. Such studies could suggest a direct role for FMR4 in specifically regulating MBD4 and gene expression more broadly, although this cannot be concluded definitively based solely on changes in mRNA levels. We acknowledge that differences in RNA processing or any number of effects downstream of FMR4 could be responsible for the observed differential gene expression, therefore it would be useful to examine precise interactions between FMR4 and its targets. Chromatin immunoprecipitation experiments could, for example, establish an interaction between FMR4 and promoter regions of target genes. Based on the function of other lncRNAs, one could speculate that 
FMR4 helps form three-dimensional interactions between distant sequence elements such as promoters and enhancers (Ma et al., 2014), and that FMR4 polyadenylation targets this transcript for further RNA processing (Norbury, 2013). Answering these questions would help our understanding of FMR4 function in particular and continue the rapid expansion of evidence on the elements that govern lncRNA actions in general.

In this study we have described novel facets of FMR4 functionality as it relates to neurodevelopment, and suggest that perturbation in the expression of this lncRNA may contribute to pathogenesis of the Fragile $\mathrm{X}$ repeat expansionassociated disorders. We report that trans-regulatory activity of FMR4 is corroborated by changes in target gene expression during differentiation of hNPCs. With this new information, we have further developed the evidence supporting the role of primate-specific lncRNAs in complex developmental programs and opened new avenues of research into the causes and therapies for Fragile $\mathrm{X}$-associated neurological disorders.

\section{Funding}

This work was supported by the National Institutes of Health (MH084880 to CW, HD057521 to VL and NS059866 to JB), as

\section{References}

Ballestar, E., and Wolffe, A. P. (2001). Methyl-CpG-binding proteins. Targeting specific gene repression. Eur. J. Biochem. 268, 1-6. doi: 10.1046/j.14321327.2001.01869.x

Banfai, B., Jia, H., Khatun, J., Wood, E., Risk, B., Gundling, W. E., et al. (2012). Long noncoding RNAs are rarely translated in two human cell lines. Genome Res. 22, 1646-1657. doi: 10.1101/gr.134767.111

Benes, F. M., Lim, B., and Subburaju, S. (2009). Site-specific regulation of cell cycle and DNA repair in post-mitotic GABA cells in schizophrenic versus bipolars. Proc. Natl. Acad. Sci. U.S.A. 106, 11731-11736. doi: 10.1073/pnas.09030 66106

Cheng, J., Kapranov, P., Drenkow, J., Dike, S., Brubaker, S., Patel, S., et al. (2005). Transcriptional maps of 10 human chromosomes at 5nucleotide resolution. Science 308, 1149-1154. doi: 10.1126/science. 1108625

Coffee, B., Zhang, F., Ceman, S., Warren, S. T., and Reines, D. (2002). Histone modifications depict an aberrantly heterochromatinized FMR1 gene in fragile $\mathrm{x}$ syndrome. Am. J. Hum. Genet. 71, 923-932. doi: 10.1086/342931

Coffee, B., Zhang, F., Warren, S. T., and Reines, D. (1999). Acetylated histones are associated with FMR1 in normal but not fragile X-syndrome cells. Nat. Genet. 22, 98-101. doi: 10.1038/8807

De Smith, A. J., Purmann, C., Walters, R. G., Ellis, R. J., Holder, S. E., Van Haelst, M. M., et al. (2009). A deletion of the HBII-85 class of small nucleolar RNAs (snoRNAs) is associated with hyperphagia, obesity and hypogonadism. Hum. Mol. Genet. 18, 3257-3265. doi: 10.1093/hmg/ddp263

Djebali, S., Davis, C. A., Merkel, A., Dobin, A., Lassmann, T., Mortazavi, A., et al. (2012). Landscape of transcription in human cells. Nature 489, 101-108. doi: 10.1038 /nature 11233

Duszczyk, M. M., Wutz, A., Rybin, V., and Sattler, M. (2011). The Xist RNA A-repeat comprises a novel AUCG tetraloop fold and a platform for multimerization. RNA 17, 1973-1982. doi: 10.1261/rna.2747411

Fabian, M. R., and Sonenberg, N. (2012). The mechanics of miRNA-mediated gene silencing: a look under the hood of miRISC. Nat. Struct. Mol. Biol. 19, 586-593. doi: $10.1038 /$ nsmb.2296 well as the Lois Pope LIFE Foundation Development Award and the Louis J. Elsas Research Award in Biochemical Genetics to VP.

\section{Author Contributions}

VP, CP, DM, KW, DV, and MM contributed to the acquisition, analysis, and interpretation of data. VP, CP, ZZ, JB, VL, JS, and $\mathrm{CW}$ made substantial contributions to the conception and design of the work. All authors participated in drafting and/or revising the manuscript for intellectual content, approval for publication and agree to be accountable for all aspects of the work.

\section{Acknowledgments}

The authors would like to acknowledge Yan Shi, MS, for her help with high content screening assays and Nagi Ayad, Ph.D., for his guidance and critical reading of the manuscript.

\section{Supplementary Material}

The Supplementary Material for this article can be found online at: http://journal.frontiersin.org/article/10.3389/fgene. 2015.00263

Faghihi, M. A., Modarresi, F., Khalil, A. M., Wood, D. E., Sahagan, B. G., Morgan, T. E., et al. (2008). Expression of a noncoding RNA is elevated in Alzheimer's disease and drives rapid feed-forward regulation of beta-secretase. Nat. Med. 14, 723-730. doi: 10.1038/nm1784

Gutschner, T., Hammerle, M., and Diederichs, S. (2013). MALAT1 - a paradigm for long noncoding RNA function in cancer. J. Mol. Med. (Berl.) 91, 791-801. doi: 10.1007/s00109-013-1028-y

Hajjari, M., Khoshnevisan, A., and Shin, Y. K. (2014). Molecular function and regulation of long non-coding RNAs: paradigms with potential roles in cancer. Tumour Biol. 35, 10645-10663. doi: 10.1007/s13277-014-2636-Z

Hornstra, I. K., Nelson, D. L., Warren, S. T., and Yang, T. P. (1993). High resolution methylation analysis of the FMR1 gene trinucleotide repeat region in fragile X syndrome. Hum. Mol. Genet. 2, 1659-1665. doi: 10.1093/hmg/2.10. 1659

Hung, T., Wang, Y., Lin, M. F., Koegel, A. K., Kotake, Y., Grant, G. D., et al. (2011). Extensive and coordinated transcription of noncoding RNAs within cell-cycle promoters. Nat. Genet. 43, 621-629. doi: 10.1038/ng.848

Kenneson, A., Zhang, F., Hagedorn, C. H., and Warren, S. T. (2001). Reduced FMRP and increased FMR1 transcription is proportionally associated with CGG repeat number in intermediate-length and premutation carriers. Hum. Mol. Genet. 10, 1449-1454. doi: 10.1093/hmg/10.14.1449

Khalil, A. M., Faghihi, M. A., Modarresi, F., Brothers, S. P., and Wahlestedt, C. (2008). A novel RNA transcript with antiapoptotic function is silenced in fragile X syndrome. PLoS ONE 3:e1486. doi: 10.1371/journal.pone.0001486

Kino, T., Hurt, D. E., Ichijo, T., Nader, N., and Chrousos, G. P. (2010). Noncoding RNA gas5 is a growth arrest- and starvation-associated repressor of the glucocorticoid receptor. Sci. Signal. 3:ra8. doi: 10.1126/scisignal.2000568

Kondo, E., Gu, Z., Horii, A., and Fukushige, S. (2005). The thymine DNA glycosylase MBD4 represses transcription and is associated with methylated p16(INK4a) and hMLH1 genes. Mol. Cell. Biol. 25, 4388-4396. doi: 10.1128/MCB.25.11.4388-4396.2005

Kotake, Y., Nakagawa, T., Kitagawa, K., Suzuki, S., Liu, N., Kitagawa, M., et al. (2011). Long non-coding RNA ANRIL is required for the PRC2 recruitment to and silencing of p15(INK4B) tumor suppressor gene. Oncogene 30, 1956-1962. doi: 10.1038/onc.2010.568 
Kumari, D., and Usdin, K. (2010). The distribution of repressive histone modifications on silenced FMR1 alleles provides clues to the mechanism of gene silencing in fragile X syndrome. Hum. Mol. Genet. 19, 4634-4642. doi: $10.1093 / \mathrm{hmg} / \mathrm{ddq} 394$

Ladd, P. D., Smith, L. E., Rabaia, N. A., Moore, J. M., Georges, S. A., Hansen, R. S., et al. (2007). An antisense transcript spanning the CGG repeat region of FMR1 is upregulated in premutation carriers but silenced in full mutation individuals. Hum. Mol. Genet. 16, 3174-3187. doi: 10.1093/hmg/ddm293

Ma, W., Ay, F., Lee, C., Gulsoy, G., Deng, X., Cook, S., et al. (2014). Finescale chromatin interaction maps reveal the cis-regulatory landscape of human lincRNA genes. Nat. Methods 12, 71-78. doi: 10.1038/nmeth.3205

Mercer, T. R., Qureshi, I. A., Gokhan, S., Dinger, M. E., Li, G., Mattick, J. S., et al. (2010). Long noncoding RNAs in neuronal-glial fate specification and oligodendrocyte lineage maturation. BMC Neurosci. 11:14. doi: 10.1186/14712202-11-14

Miyagawa, R., Tano, K., Mizuno, R., Nakamura, Y., Ijiri, K., Rakwal, R., et al. (2012). Identification of cis- and trans-acting factors involved in the localization of MALAT-1 noncoding RNA to nuclear speckles. RNA 18, 738-751. doi: 10.1261/rna.028639.111

Norbury, C. J. (2013). Cytoplasmic RNA: a case of the tail wagging the dog. Nat. Rev. Mol. Cell Biol. 14, 643-653. doi: 10.1038/nrm3645

Oostra, B. A., and Willemsen, R. (2003). A fragile balance: FMR1 expression levels. Hum. Mol. Genet. 12, R249-R257. doi: 10.1093/hmg/ddg298

Pastori, C., Peschansky, V. J., Barbouth, D., Mehta, A., Silva, J. P., and Wahlestedt, C. (2014). Comprehensive analysis of the transcriptional landscape of the human FMR1 gene reveals two new long noncoding RNAs differentially expressed in Fragile $\mathrm{X}$ syndrome and Fragile $\mathrm{X}$-associated tremor/ataxia syndrome. Hum. Genet. 133, 59-67. doi: 10.1007/s00439-013-1356-6

Powell, W. T., Coulson, R. L., Crary, F. K., Wong, S. S., Ach, R. A., Tsang, P., et al. (2013). A Prader-Willi locus lncRNA cloud modulates diurnal genes and energy expenditure. Hum. Mol. Genet. 22, 4318-4328. doi: 10.1093/hmg/ddt281

Qureshi, I. A., Mattick, J. S., and Mehler, M. F. (2010). Long non-coding RNAs in nervous system function and disease. Brain Res. 1338, 20-35. doi: 10.1016/j.brainres.2010.03.110

Rinn, J. L., Kertesz, M., Wang, J. K., Squazzo, S. L., Xu, X., Brugmann, S. A., et al. (2007). Functional demarcation of active and silent chromatin domains in human HOX loci by noncoding RNAs. Cell 129, 1311-1323. doi: 10.1016/j.cell.2007.05.022

Rother, S., and Meister, G. (2011). Small RNAs derived from longer non-coding RNAs. Biochimie 93, 1905-1915. doi: 10.1016/j.biochi.2011.07.032

Ruddock-D'cruz, N. T., Xue, J., Wilson, K. J., Heffernan, C., Prashadkumar, S., Cooney, M. A., et al. (2008). Dynamic changes in the localization of five members of the methyl binding domain (MBD) gene family during murine and bovine preimplantation embryo development. Mol. Reprod. Dev. 75, 48-59. doi: $10.1002 / \mathrm{mrd} .20712$

Sahoo, T., Del Gaudio, D., German, J. R., Shinawi, M., Peters, S. U., Person, R. E., et al. (2008). Prader-Willi phenotype caused by paternal deficiency for the HBII-85 C/D box small nucleolar RNA cluster. Nat. Genet. 40, 719-721. doi: $10.1038 /$ ng. 158
Smyth, G. K. (2004). Linear models and empirical bayes methods for assessing differential expression in microarray experiments. Stat. Appl. Genet. Mol. Biol. 3, 3. doi: 10.2202/1544-6115.1027

Sopher, B. L., Ladd, P. D., Pineda, V. V., Libby, R. T., Sunkin, S. M., Hurley, J. B., et al. (2011). CTCF regulates ataxin-7 expression through promotion of a convergently transcribed, antisense noncoding RNA. Neuron 70, 1071-1084. doi: 10.1016/j.neuron.2011.05.027

Sutcliffe, J. S., Nelson, D. L., Zhang, F., Pieretti, M., Caskey, C. T., Saxe, D., et al. (1992). DNA methylation represses FMR-1 transcription in fragile X syndrome. Hum. Mol. Genet. 1, 397-400. doi: 10.1093/hmg/1.6.397

Tassone, F., Hagerman, R. J., Taylor, A. K., Gane, L. W., Godfrey, T. E., and Hagerman, P. J. (2000). Elevated levels of FMR1 mRNA in carrier males: a new mechanism of involvement in the fragile-X syndrome. Am. J. Hum. Genet. 66, 6-15. doi: 10.1086/302720

Tsai, M. C., Manor, O., Wan, Y., Mosammaparast, N., Wang, J. K., Lan, F., et al. (2010). Long noncoding RNA as modular scaffold of histone modification complexes. Science 329, 689-693. doi: 10.1126/science.1192002

Velmeshev, D., Magistri, M., and Faghihi, M. A. (2013). Expression of nonprotein-coding antisense RNAs in genomic regions related to autism spectrum disorders. Mol. Autism 4, 32. doi: 10.1186/2040-2392-4-32

Wang, K. C., and Chang, H. Y. (2011). Molecular mechanisms of long noncoding RNAs. Mol. Cell. 43, 904-914. doi: 10.1016/j.molcel.2011.08.018

Yap, K. L., Li, S., Munoz-Cabello, A. M., Raguz, S., Zeng, L., Mujtaba, S., et al. (2010). Molecular interplay of the noncoding RNA ANRIL and methylated histone $\mathrm{H} 3$ lysine 27 by polycomb CBX7 in transcriptional silencing of INK4a. Mol. Cell. 38, 662-674. doi: 10.1016/j.molcel.2010.03.021

Zhang, L. J., Zhu, Y. N., Gao, Y., Liu, S. Y., Zhai, B., Li, C. H., et al. (2014a). The MBD4 gene plays an important role in porcine adipocyte differentiation. Cell Physiol. Biochem. 34, 1216-1226. doi: 10.1159/000366333

Zhang, Y., Yang, L., and Chen, L. L. (2014b). Life without A tail: new formats of long noncoding RNAs. Int. J. Biochem. Cell Biol. 54, 338-349. doi: 10.1016/j.biocel.2013.10.009

Ziats, M. N., and Rennert, O. M. (2013). Aberrant expression of long noncoding RNAs in autistic brain. J. Mol. Neurosci. 49, 589-593. doi: 10.1007/s12031-0129880-8

Conflict of Interest Statement: The reviewer Guney Bademci declares that, despite being affiliated with the same institute as the authors Veronica J. Peschansky, Chiara Pastori, Zane Zeier, Dario Motti, Katya Wentzel, Dmitry Velmeshev, Marco Magistri, John L. Bixby, Vance P. Lemmon, José P. Silva, and Claes Wahlestedt, the review process was conducted objectively.

Copyright (C) 2015 Peschansky, Pastori, Zeier, Motti, Wentzel, Velmeshev, Magistri, Bixby, Lemmon, Silva and Wahlestedt. This is an open-access article distributed under the terms of the Creative Commons Attribution License (CC BY). The use, distribution or reproduction in other forums is permitted, provided the original author(s) or licensor are credited and that the original publication in this journal is cited, in accordance with accepted academic practice. No use, distribution or reproduction is permitted which does not comply with these terms. 